\title{
Study on Shadow Processing Method Based on Background of Matlab Scans
}

\author{
Zhao Jian-fei, Zhang xiao-juan \\ Qinghai Normal University, Xining, China \\ zhao12866458@163.com
}

\section{Keywords: Matlab scanning shadow image processing}

\begin{abstract}
In the process of constructing Dongxiang nationality literature and data database, some ancient books and materials were scanned into scans. Due to the long age, the background of scanned parts was severely affected and the readability of scans was affected. This paper uses the image processing function of Matlab software. The scanned parts are preprocessed and new image processing methods are adopted, which greatly improves the readability of the scanned parts, and plays an optimal role in the construction of the Dongxiang national literature database.
\end{abstract}

\section{Introduction}

MATLB $^{[1]}$ is a kind of language called "my grass paper type scientific computing language", it is in the numerical calculation, data processing, automatic control, signal processing, neural network, optimization, fuzzy logic, wavelet analysis, image processing, statistical analysis, financial analysis and many other fields have a wide range of uses. In particular, the various toolboxes it provides enables us to do all kinds of computation, calculus and Simulation in scientific computing, engineering design, numerical analysis and image processing. The MATLAB image processing toolbox supports four basic image types: the index image, the gray image, the binary image and the RGB image. The image that MATLAB reads directly from the image file is a RGB image. It is stored in a three-dimensional array. The three dimensional array has three faces, which correspond to three colors of red (Red), green (Green) and blue (Blue). The data in the surface are three color intensity values. The elements in the surface correspond to pixels in the image.

Image enhancement is one of the most important research problems in image processing. Image enhancement can be divided into two categories according to the scope of action, namely, space processing and frequency domain processing. The spatial processing is directly processing the image, while the frequency domain processing is based on the transformation coefficient of the image in a changing area 
of the image, and then obtains the image enhancement effect by inverse transformation. Image enhancement can highlight some of the "useful" information in the image, expand the difference between the features of different objects in the image, and improve the visual effect of the image. Image enhancement algorithms include histogram enhancement, spatial filtering enhancement, frequency domain filtering enhancement, and color enhancement.

Image restoration (recovery) inevitably causes the degradation of image quality due to the leakage and dislocation of the scanning line caused by the equipment. Image restoration is based on the established system degradation model in advance, and the degraded image is reconstructed to the original ideal image which is close to or completely degenerate.

\section{Image type}

2.1、 Bitmap, pixel map, raster graph, image, dot matrix ${ }^{[2]}$, these names are described in the same type, because these names indicate that they are made up of pixels. These pixels can be arranged and dyed to form a pattern. When the bitmap is magnified, you can see countless single squares that make up the entire image. The effect of enlarging the size of the bitmap is to increase the single pixel, so that the lines and shapes are uneven. However, if you watch it from a bit far away, the color and shape of the bitmap image is continuous. Because each pixel is individually stained, it can produce realistic effects of similar photos by selecting the region at the frequency of one pixel, such as deepening the shadow and increasing the color. Reducing the size of the bitmap will also deform the original, because it reduces the whole image by reducing the pixels.

2.2 The lattice image is related to the resolution, that is, a fixed number of pixels are contained on a certain area of the image. Therefore, if the image is displayed on the screen with large multiple magnification, or at a low resolution, the bitmap image will have a serrated edge.

\section{Matlab steps for processing images}

\subsection{Read and display images, as shown in Figure 1}

>> clear;close all

>> I=imread('rice.png');

$>$ imshow $(\mathrm{I})$ 


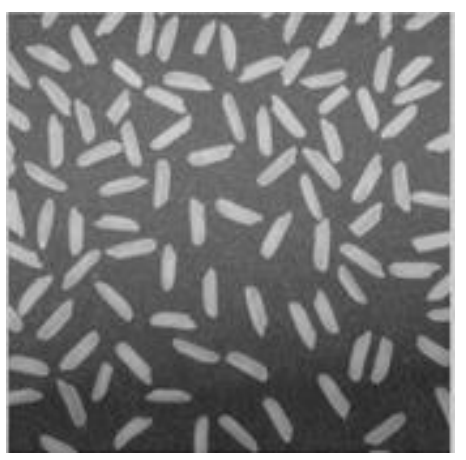

Figure 1

\subsection{Estimation of image background ${ }^{[3]}$}

The center location and brightness of the image are stronger than those of other parts. Using imopen function and a circular structure element with a radius of 15 , we do morphological operations on the input image I, and remove the objects that are not included in the disc, so that we can estimate the background brightness.

> clear;close all

> I=imread('rice.png');

$>$ imshow $(\mathrm{I})$

>> background=imopen(I,strel('disk',15));

> imshow(background)

> figure,surf(double(background(1:8:end,1:8:end))),zlim([0,255]);

> set(gca,'ydir','reverse');

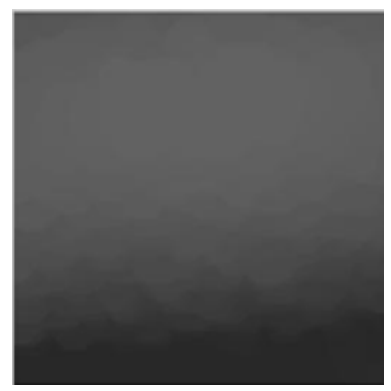

Figure 2

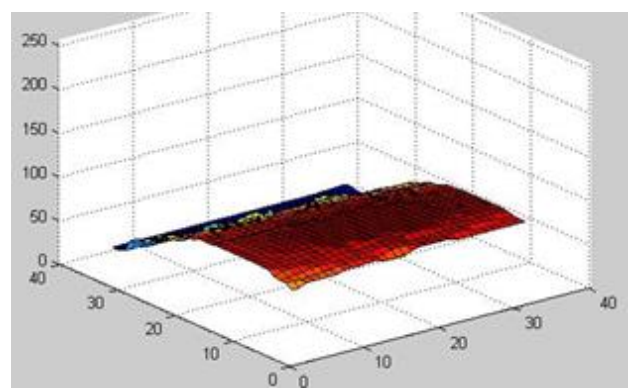

Figure 3

\section{MatlabOptimize the image}

4.1. The background image ${ }^{[4]}$ is subtracted from the original image (the original image I subtracts the background image to get a more consistent background image):

>> I2=imsubtract(I,background);

> figure,imshow(I2)

4.2. Modulate the contrast of the image ${ }^{[5]}$ (the image is dark, using the imadjust function command to adjust the contrast of the image)

>> I3=imadjust(I2,stretchlim(I2),[0 1]);

> figure,imshow(I3); 


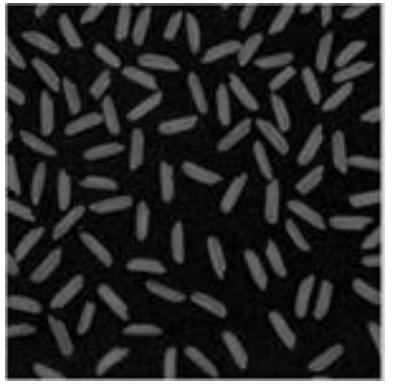

Figure 4

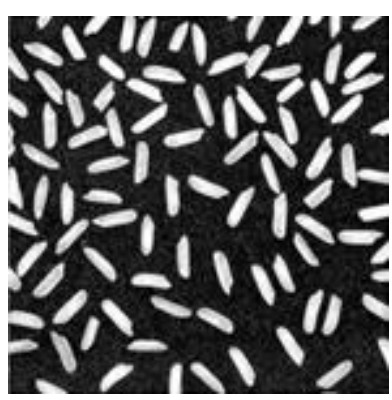

Figure 5

\subsection{Other methods}

Wiener filter belongs to modern filter ${ }^{[6]}$ filter can only eliminate the signal and interference frequency band of traditional non overlapping, when the signal and interference band overlap when traditional filters will be incapable of action, it is necessary to use modern filter, modern filter by using the statistical characteristics of signal and interference (such as self power spectrum correlation function, etc.) are a the optimal estimation algorithm, and then realized by hardware or software. Wiener filtering is based on the minimum mean square error (LMS (Least MeanSquare) criterion. It estimates the current value of signals based on past observations and current observations, so the solution form is the transfer function or unit impulse response of the system. The mean square error is: $E\left[e^{2}(n)\right]=E\left[(s(n) \hat{s}(n))^{2}\right]$ 。

The parameters of the Wiener filter are fixed, and are suitable for stationary random signals. The Calman filter parameters are time-varying and are suitable for non-stationary random signals. However, only when the statistical characteristics of the signal and noise are known prior to the two filtering techniques can obtain the optimal filtering. In practical applications, it is often impossible to obtain prior knowledge of the statistical characteristics of signal and noise. In this case, adaptive filtering technology can obtain excellent filtering performance, so it has good application value.

\section{Conclusion}

Through the above methods, the image processing function of Matlab is applied to scan the processed parts, and achieved satisfactory results, thus greatly improving the readability of the scanning parts, and optimizing the construction of the literature database.

\section{Acknowledgment}

This work is supported by National Social Science Fund Project "Research on Construction of Dongxiang Ethnic Literature Database" (15XTQ007); National Social Science Fund Project "Qinghai Embroidery Art Digital Dictionary Resource Bank Construction" (15XMZ057); Qinghai Provincial Science and Technology Department Applied Basic Research Project "Based on 3D scanning and Research on Digital 
Acquisition and Inheritance Learning Technology of Qinghai Embroidery Art by Virtual Hand (2015-ZJ-718), all support is gratefully acknowledged.

\section{References}

[1] MATLAB based image processing and analysis [J]. He Xiping. Journal of Industrial and Commercial University Of Chongqing (NATURAL SCIENCE EDITION). 2003 (02)

[2] image fusion algorithm based on the contrast of multi wavelet domain [J]. Wang Zhihui. System engineering and electronic technology. 2008 (10)

[3] Li Guoyong. Intelligent control and its MATLAB implementation [M].2 edition. Electronic Industry Press, 2005.

[4] Wang Qiang. Using artificial neural network to realize function approximation to [J]. computer simulation, 2002, (9): 44-46.

[5] using MATLAB for digital image analysis and processing [J]. Huang Jianling. Computer and modernization. 2000 (06)

[6] Looney C G. Advances in Feedforward Neural Networks, Denystifying Knowledge Acquiring Black Baxes. IEEE Trans Knowledge and Data Engeneering, 1996, 8: 211-226. 